\section{Medial floor plate formation in zebrafish consists of two phases and requires trunk-derived Midkine-a}

\author{
Matthias Schäfer, ${ }^{1}$ Martina Rembold, ${ }^{2}$ \\ Joachim Wittbrodt, ${ }^{2}$ Manfred Schartl, ${ }^{1}$ and \\ Christoph Winkler ${ }^{1,3}$ \\ ${ }^{1}$ Department of Physiological Chemistry I, Biocenter \\ University of Würzburg, Würzburg 97074, Germany; \\ ${ }^{2}$ European Molecular Biology Laboratory, Heidelberg \\ 69117, Germany
}

The medial floor plate (MFP) organizes the specification of neurons and outgrowth of axons in the ventral spinal cord of vertebrates. We show that the growth factor Midkine-a, expressed in the paraxial mesoderm, is required for formation of the MFP in zebrafish. Our epistatic analyses demonstrate that development of MFP comprises two independent sequential phases. Following initial MFP induction in the gastrula organizer, Midkine-a regulates allocation of MFP cells during subsequent development. Thus in zebrafish, trunk-derived signals are required for complete MFP formation from a common pool of organizer-derived midline precursor cells.

Supplemental material is available at http://www.genesdev.org.

Received July 1, 2004; revised version accepted February 28, 2005.

The medial floor plate (MFP) is the ventral-most structure in the vertebrate neural tube with important organizer functions during specification and differentiation of neurons and glia. Its origin and the timing of the specification are matters of ongoing debates (see Strähle et al. 2004). In zebrafish, mutant analyses and lineage tracing have shown that MFP cells originate from a pool of midline precursor cells in the gastrula organizer, the embryonic shield (Appel et al. 1999; Le Douarin and Halpern 2000). Accordingly, the induction of the MFP in zebrafish occurs during gastrulation by shield-derived factors (Tian et al. 2003). Initially induced MFP precursors persist in the tailbud and contribute to the MFP during later embryonic development. The molecular mechanisms and factors that regulate allocation of cells to the MFP in the posteriorly extending trunk after gastrulation, however, remain unclear. Here we describe that midkine-a (mdka) regulates the allocation of MFP precursor cells in zebrafish during neurulation.

Midkine and the related pleiotrophin genes encode secreted heparin-binding growth factors with neurotrophic

[Keywords: neural patterning; medial floor plate; midkine; pleiotrophin; heparin-binding growth factors; zebrafish]

${ }^{3}$ Corresponding author.

E-MAIL cwinkler@biozentrum.uni-wuerzburg.de; FAX 49-931-888 4150 Article and publication are at http://www.genesdev.org/cgi/doi/10.1101/ gad.336305. activity in cell culture assays (for review, see Muramatsu 2002). In mammals, they are widely expressed during embryonic development and have been implicated in processes like outgrowth and survival of neurons, angiogenesis, wound healing, and cancer (for review, see Kadomatsu and Muramatsu 2004). In zebrafish, two midkine genes, $m d k a$ and $m d k b$ are expressed in restricted and nonoverlapping patterns during embryonic development (Winkler et al. 2003). Analyzing its expression, regulation, and activity, we show that Mdka expressed in the paraxial mesoderm functions in MFP formation in zebrafish.

\section{Results and Discussion}

mdka transcription is first detectable by RT-PCR during late gastrulation (at $75 \%$ epiboly; $8 \mathrm{~h}$ post-fertilization; hpf) (Fig. 1a). At the beginning of neurulation (10 hpf), when the tailbud starts to extend caudally to form the trunk and tail, mdka transcription is confined to the rostral paraxial mesoderm that is still unsegmented at this stage (Fig. 1b). Later, its expression moves as a wave from anterior to posterior in this region (Fig. 1c-h). The caudal front of the mdka wave in the paraxial mesoderm progresses in parallel to the formation of the neural keel that emerges from the converging neural plate (Fig. 1i,j). At the same point, the morphologically distinct MFP starts to form, which is marked by the restriction of broad tiggy-winkle hedgehog (twhh) expression to a row of single cells (Fig. 1k). Therefore, expression of $m d k a$ coincides temporally and spatially with the appearance of a morphologically recognizable floor plate. After 13 hpf, mdka expression is also found dorsally in the anterior neural tube (Fig. 11; data not shown).

We first addressed the function of mdka during MFP formation by overexpressing Mdka through mRNA injection into zebrafish embryos (Fig. 2). This resulted in a strong expansion of the MFP as defined by the expression of sonic hedgehog (shh) (Krauss et al. 1993) and other markers (Fig. 2d,e,g,h; data not shown). While the overall morphology of the embryos at the 14 somite (14 s) stage appeared normal, the width of the MFP increased from one to three cell diameters in anterior trunk regions /data not shown) and was even broader in more posterior regions (Fig. 2e,h). The effect of ubiquitously expressed mdka on the neural tube was confined to the MFP. Lateral floor plate cells expressing $n k x 2.2 b$ (Schäfer et al. 2005) and the motoneuron domain expressing olig2 (Park et al. 2002) were shifted laterally, but otherwise showed no significant differences in number and size (Fig. 21-O; see Supplementary Fig. S1). Thus, ectopic mdka specifically promotes MFP formation.

The significant increase in MFP cell number could simply be due to enhanced cell proliferation in this structure. However, immunostaining for phospho histone $\mathrm{H} 3$ and BrdU labeling to detect cells in $\mathrm{M}$ and $\mathrm{S}$ phases, respectively, showed no significant difference in proliferation rates between mdka-injected and control embryos (Supplementary Fig. S2). We also excluded the possibility of ingression of MFP precursors from neigh- 
Schäfer et al.

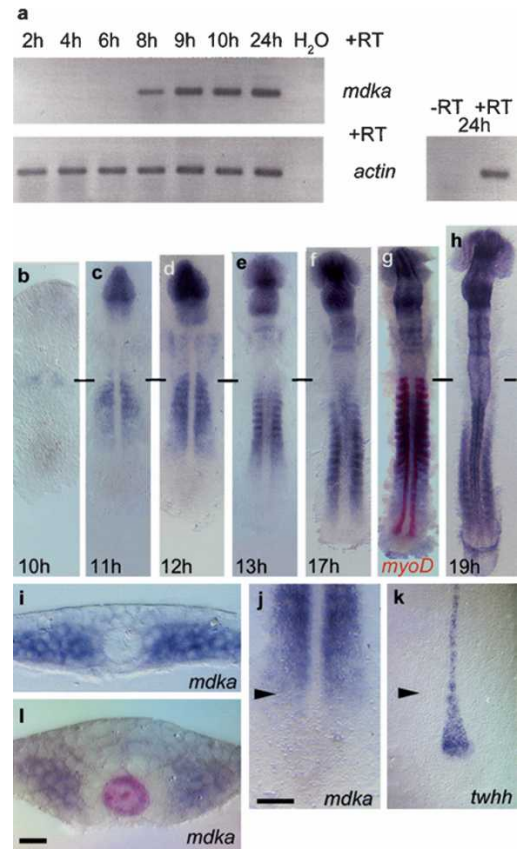

Figure 1. Mdka expression during zebrafish embryogenesis. (a) RTPCR analysis: $m d k a$ transcription is first detected at $8 \mathrm{hpf}(\mathrm{h})$; actin expression was used for calibration. -RT control using actin primers without addition of reverse transcriptase. $(b-h)$ RNA in situ hybridization on 10-19-h embryos showing advancing mdka transcription in the paraxial mesoderm; horizontal bars mark the position of the first somite. $(g)$ Double staining with myoD at $17 \mathrm{~h}$ shows progressive loss of $m d k a$ transcription in anterior somites. (i) mdka is expressed at the six-somite $(6 \mathrm{~s} ; 12 \mathrm{hpf})$ stage in cells of the unsegmented paraxial mesoderm when convergence of the neural plate has just started. $(j, k)$ The front of advancing mdka expression $(j)$ demarcates the position of $t w h h$ restriction in MFP precursors $(k)$ as indicated by arrowheads that also show level of transverse section in i. (1) At later stages, $m d k a$ is expressed in paraxial mesoderm and also the anterior neural keel, but excluded from a ring of cells surrounding the shh-positive notochord and MFP (8s stage, shh labeled in red). In dorsal views, anterior is to the top in $b-h, j, k$. Bars: $j, 10 \mu \mathrm{m} ; 1$, $50 \mu \mathrm{m}$.

boring trunk areas by using confocal time-lapse analyses (data not shown). Instead, ectopic mdka is likely to affect the specification of midline precursor cells that give rise to MFP and the notochord. This was evident by a highly significant reduction in notochord size observed in mdka RNA-injected embryos with increased MFP (Fig. $2 \mathrm{~d}-\mathrm{f}, \mathrm{k}$; Supplementary Fig. S3). More than $50 \%$ of embryos showed a complete block of notochord formation in the posterior trunk (Fig. $2 \mathrm{~g}-\mathrm{i}$ ). Ectopic mdka had no effect on axial mesoderm formation during early gastrulation ( $n=43 / 43 ; 100 \%$ of embryos with normal expression of cyclops [cyc] as an early axial mesoderm marker; data not shown) indicating that Mdka does not affect early cell specification in the shield. Rather, it impairs notochord development later during neurulation. At this stage, endogenous mdka expression is found throughout the posterior trunk, but excluded from the tailbud where notochord progenitor cells are located (Fig. 2j). This strongly argues for a role of mdka in the specification of MFP versus notochord cells within the trunk region at stages after gastrulation.

The observed formation of MFP cells at the expense of the notochord is in agreement with earlier reports on cell fate restrictions in the pool of midline precursor cells (Halpern et al. 1997; Appel et al. 1999; Amacher et al. 2002). Expansion of the MFP in zebrafish (e.g., by ectopically expressed deltaA) coincides with the reduction of the notochord (Appel et al. 1999). Also, mutants deficient for the T-box gene no tail (ntl)/Brachyury lack the notochord, and instead show a wider MFP (Halpern et al. 1997; Amacher et al. 2002). Therefore, to promote the notochord fate, $n t l$ appears to be required to repress MFP. This suggests that several counteracting pathways regulate the decision of midline cell fates. Mdka promotes MFP and acts during tailbud extension, but in contrast to $n t l$ and deltaA is not expressed in the tailbud. Conse-

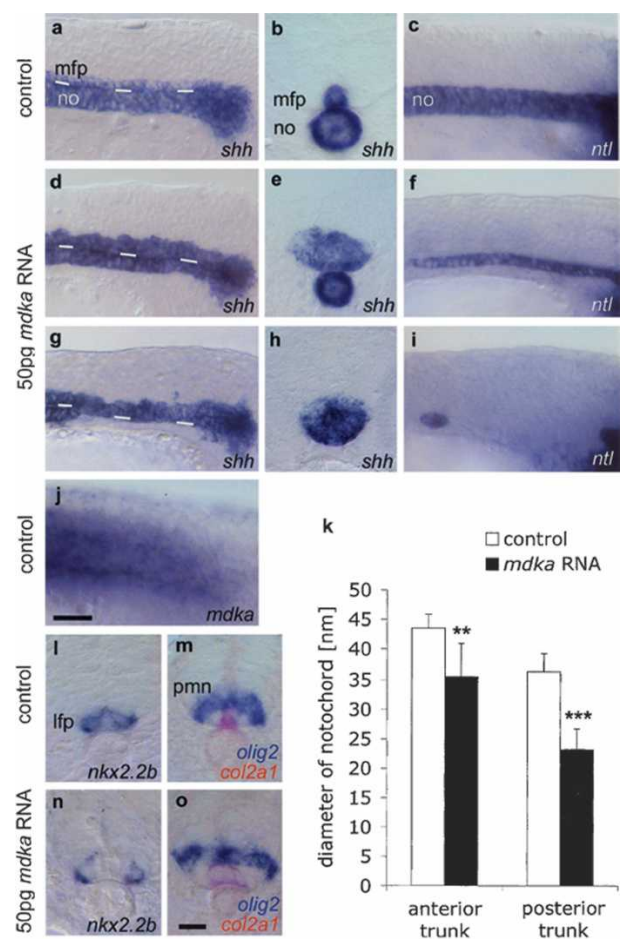

Figure 2. Mdka overexpression enlarges the MFP at the expense of the notochord. Lateral views $(a, c)$ and transverse sections $(b$, posterior trunk) of noninjected $14 \mathrm{~s}$ control embryos. $(d-f)$ In embryos injected with $50 \mathrm{pg}$ mdka RNA, the MFP is enlarged lexpanded shh in MFP, $n=165 / 191,86 \%$; $f$-spondin, in $n=36 / 46,76 \%$; col2a , $n=134 / 145,92 \%$ [data not shown]) and the notochord is reduced (reduced shh in the notochord in $\mathrm{n}=20 / 38,53 \%$; col2a1 in $n=27$ / $88,31 \%$; and $n t l$ in $n=11 / 45,24 \%) .(g-i)$ Severely affected embryo with complete block of notochord formation in the posterior trunk (lacking shh expression in $n=16 / 38,42 \%$; col2a 1 in $n=41 / 88,46 \%$, and $n t l$ in $n=30 / 45,67 \%)$. White lines in $a, d$, and $g$ indicate border between the notochord and MFP. Sections in $b, e$, and $h$ are at similar positions in the posterior trunk. (j) Endogenous mdka transcription in the paraxial mesoderm extends up to the tailbud. $(k)$ $m d k a$ overexpression resulted in a significant decrease of notochord width: $n=10 ;\left(^{\star \star}\right) p<0.01$ versus control for anterior trunk; $\left(^{\star \star \star}\right)$ $p<0.001$ versus control for posterior trunk; Mann-Whitney U-test (see Supplementary Fig. S3). (1-O) Transverse sections of 18s embryos showing enlarged MFP (expanded col2a1 in $n=37 / 44,84 \%[o]$ ), but normal expression of $n k x 2.2 b$ in the lateral floor plate $(n=27 / 29$, $93 \%[n])$ and olig2 in motoneuron domain $\left(n=43 / 44,98 \%[o]_{i}\right.$ $n=10, p=0.796$; see Supplementary Fig. S1) in mdka-injected embryos. Anterior is left in lateral views. Bars: $j, 20 \mu \mathrm{m} ; o, 10 \mu \mathrm{m}$. (lfp) Lateral floor plate, $(\mathrm{mfp})$ medial floor plate, (no) notochord, (pmn) motoneuron progenitor domain. 


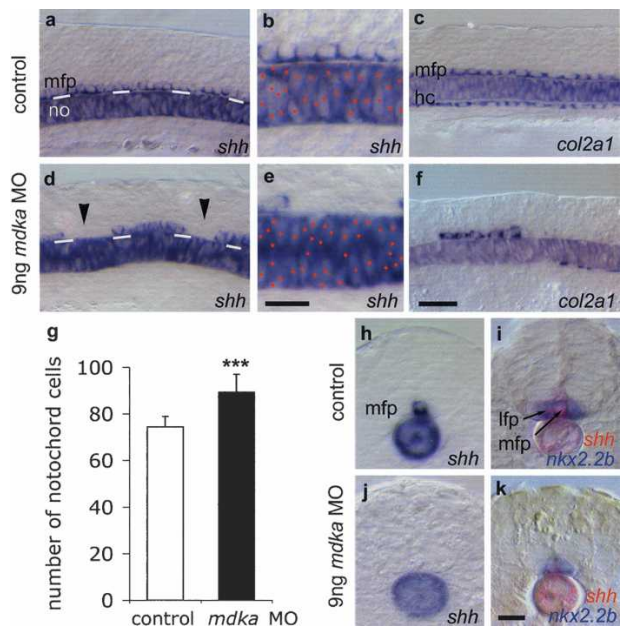

Figure 3. MFP formation requires Mdka protein. Lateral views of noninjected control $(a-c)$ and 9 ng mdka MO morpholino-injected 12s embryos $(d-f)$. mdka MO-injected embryos show gaps in the MFP (arrowheads; reduced MFP expression of shh in $n=103 / 136$, $76 \%$ of injected embryos $[d, e]$; col2a1 in $n=114 / 188,61 \%[f]$; and $f$-spondin in $n=19 / 30,63 \%$ [data not shown]) and increased cell density in the notochord (expressing shh $[d, e]$ and $n t l, n=17 / 27$, $63 \%$ [data not shown]). White lines in $a$ and $d$ indicate the border between the notochord and MFP. Red dots mark notochord cells in $b$ and $e$. Large gaps were also observed in the hypochord (col2a1 reduced in $n=146 / 188,78 \%$ of embryos $[f]) .(g)$ Mdka MO significantly increased the number of notochords cells: $n=10^{;}\left(^{\star \star \star}\right)$ $p<0.001$ versus control; Mann-Whitney U-test (see Supplementary Fig. S5). (h-k) Cross-sections of control $(h, i)$ and mdka MO-injected $(j, k)$ embryos at the trunk level showing loss of shh-positive MFP cells and fusion of $n k x 2.2 b$-expressing lateral floor plate cells (in $n=13 / 22,59 \%$ of embryos). In several cases $(n=9 / 22,41 \%)$ also lateral floor plate cells were missing, possibly due to insufficient levels of Shh. Bar: $f, 20 \mu \mathrm{m} ; b, k, 10 \mu \mathrm{m}$. (hc) hypochord.

quently, inactivation of Mdka should therefore lead to a reduction of MFP during post-gastrulation stages.

To inactivate Mdka function we used a morpholino antisense approach (Nasevicius and Ekker 2000). Knockdown of $m d k a$ resulted in severe defects in MFP formation (Fig. 3). Analysis of several markers revealed large and irregularly spaced gaps in the MFP (Fig. 3d-f). These were consistently restricted to the trunk region posterior to the hindbrain, supporting the hypothesis that $m d k a$ is not involved in MFP formation during early gastrulation. We never obtained a complete lack of MFP in morpholino-injected embryos. This indicates that parallel pathways regulate MFP formation. A similar situation was reported in Delta-Notch-deficient mutant embryos (Appel et al. 1999), while mutants deficient for the nodalrelated gene cyc show a complete loss of MFP (Halpern et al. 1997; Tian et al. 2003). The appearance of gaps is best explained by an incomplete redundancy of these pathways in different precursor cell populations. Alternatively, these gaps could reflect an incomplete knockdown of Mdka that results in an uneven allocation of remaining MFP cells during tailbud extension movements.

Interestingly, in the floor plate, the effect of Mdka depletion is specific for the medial cells. Lateral floor plate cells form normally, but collapse in the midline (Fig. 3h-k). Importantly, in the majority of embryos, we observed a highly significant increase in cell density in the notochord (Fig. 3d,e,g; Supplementary Fig. S5). This suggests that in contrast to gain of function, in the absence of Mdka protein notochord formation is promoted at the expense of MFP. Further supporting the idea that MFP, notochord, and hypochord cells originate from a common pool of midline precursor cells (Halpern et al. 1997; Appel et al. 1999) and that mdka is required for allocation of initially induced progenitor cells, we also
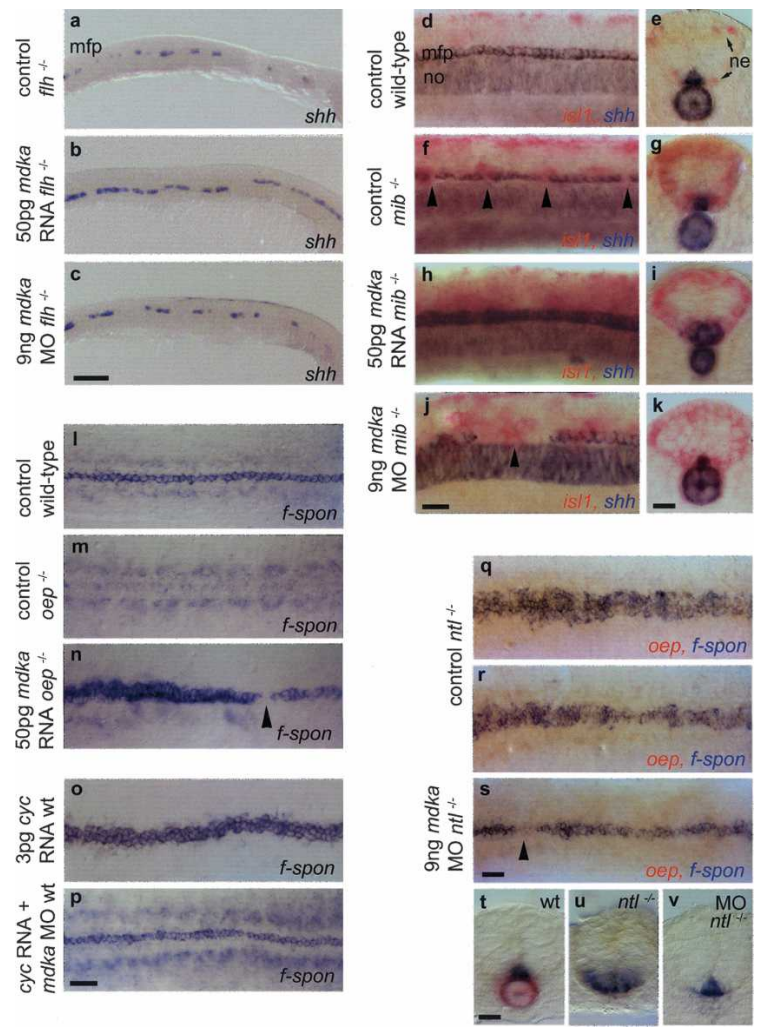

Figure 4. Epistatic interactions of mdka. $(a, c)$ In floating head mutants $\left(f 1 h^{-1-}\right)$ that lack a notochord and only retain islands of MFP cells $(a), m d k a$ overepression partially rescues the MFP defect. $(a, b)$ Cell number is increased, resulting in an almost continuous line one cell in diameter $(n=21 / 27,78 \%)$. Knockdown has no effect on MFP cell number and does not rescue the notochord $(n=42$, shh $[c]$; $n=18, n t$ [ [data not shown]). $(d-k)$ Delta/Notch-deficient mindbomb mutants $\left(\mathrm{mib}^{-/-}\right)$lack MFP cells $(f)$ and were identified by an increase in isl1-positive neurons (ne, in red $[g, i, k]) .(h-k) M d k a$ overexpression and knockdown produces identical MFP and notochord phenotypes in $\mathrm{mib}^{-/-}$as in wild-type embryos. Overexpression enlarges the MFP $(n=11 / 15,73 \%[h, i])$ and knockdown results in MFP gaps larger than in noninjected $\mathrm{mib}^{-/-}(n=11 / 18,61 \%[j])$. (l-n) oneeyed pinhead $\left(\mathrm{oep}^{-1-}\right)$ embryos that lack the entire MFP $(\mathrm{m})$ were rescued by injection of maka RNA $(n=12 / 14,86 \%[n])$. In these embryos, the broadness of the MFP was increased. oep ${ }^{-/}$were identified by remaining gaps in the $f$-spondin-positive MFP (arrowhead). $(o, p)$ Overexpression of cyclops (cyc) RNA in wild-type embryos resulted in an enlargement of the MFP from one to three cells in width $(n=36 / 57,63 \%[o])$. Coinjection of $c y c$ RNA and mdka morpholino resulted in a MFP with nearly wild-type morphology $(n=73 / 87,83 \%[p]) .(q-V)$ In no-tail mutants $\left(n t 7^{-1-}\right)$, MFP width is enlarged to variable extents $(q, r, u)$, but is nearly rescued after $m d k a$ morpholino injection $(s, V)$. ntt $1^{-/}$embryos were identified by the absence of a notochord and oep expression $(q-s, u, v)$. mdka knockdown is not able to rescue the notochord. Anterior is to the left in lateral $(a-c, d, f, h, j)$ and dorsal $(l-s)$ views; arrowheads indicate gaps in MFP. $(e, g, i, k, t-V)$ Transverse sections at trunk level. Bars: $c, 100 \mu \mathrm{m}$; $p, s, 40 \mu \mathrm{m} ; i, k, t, 20 \mu \mathrm{m}$. 
noticed defective hypochord development in mdka morpholino-injected embryos. Large gaps were evident in this structure located underneath the notochord (Fig. 3f).

Cell lineage and mutant analyses in chicken, Xenopus, and zebrafish suggested that MFP specification occurs early during gastrulation in the organizer (Halpern et al. 1997; Teillet et al. 1998; Appel et al. 1999; Lopez et al. 2003). In zebrafish, the continuous signaling by nodalrelated Cyclops is required for early MFP induction during gastrulation (Tian et al. 2003). In addition, Ntl, Floating head (Flh) and the Delta-Notch pathway are implicated in MFP formation (Halpern et al. 1997; Appel et al. 1999; Amacher et al. 2002). As Mdka promotes MFP at the expense of the notochord, our data provide additional support for this model and open the possibility that Mdka interacts with these pathways during allocation of MFP cells initially induced in the shield. To investigate Mdka's interactions with these factors, we analyzed the effects of $m d k a$ overexpression and knockdown in several mutant conditions (Fig. 4).

Floating head $\left(f 1 h^{-/-}\right)$mutants lack the notochord completely, and only retain islands of MFP cells (Fig. 4a; Halpern et al. 1997). Ectopic expression of $m d k a$ in $f 1 h^{-/-}$ mutants partially rescued the MFP defect. On the other hand, knockdown had no influence on the remaining MFP cells, suggesting that endogenous Mdka is not responsible for the appearance of remaining MFP islands in $\mathrm{flh}^{-/-}$mutants (Fig. 4b,c). Mindbomb (mib) mutants are deficient for a RING-type ubiquitin ligase that is essential for Delta/Notch signaling (Itoh et al. 2003). These mutants lack single MFP cells similar to the situation in $d l A^{-/-}$ mutants (Fig. 4f; Appel et al. 1999). Mdka overexpression and knockdown in $\mathrm{mib}^{-/-}$mutants produced identical MFP and notochord phenotypes as in wild-type embryos (Fig. 4h-i). This suggests that the Mdka pathway acts in parallel to and independent of Delta/Notch signaling.

Mutant analyses in zebrafish have shown that continuous Cyc signaling during 60\%-90\% epiboly is crucial for MFP formation (Tian et al. 2003). One-eyed-pinhead (oep) encodes an essential extracellular cofactor for Cyc signaling (Gritsman et al. 1999). Homozygous oep mutants consequently lack the complete MFP (Fig. 4m; Strähle et al. 1997). Overexpression of $m d k a$ in oep ${ }^{-/-}$ mutants rescued the mutant phenotype and resulted in an enlarged MFP (Fig. 4n). To show that mdka is also required for $c y c$ dependent MFP formation, we coinjected $c y c$ RNA together with mdka morpholinos. Overexpression of $c y c$ alone resulted in an expansion of MFP (Fig. 40; Tian et al. 2003). Coinjection rescued this effect and resulted in a MFP with normal width and sporadic appearance of gaps (Fig. 4p; data not shown), suggesting that $m d k a$ is required for the effects of $c y c$ overexpression on MFP expansion. $\mathrm{Nt}^{-/-}$mutants, as described above, lack a notochord, but have an enlarged MFP (Fig. $4 \mathrm{q}, \mathrm{r}, \mathrm{u})$. In $n t 1^{-/-}$, mdka knockdown resulted in a nearly complete rescue of the MFP phenotype (Fig. 4s,v). However, no rescue of the notochord was observed, possibly due to defects in the earliest steps of notochord formation. This shows that mdka affects MFP cells even in the absence of a notochord. Taken together, these results indicate that $m d k a$ is capable of rescuing defects in early MFP induction.

Intriguingly, mdka overexpression could rescue MFP formation in mutants with defects in early MFP induction, which show reduced number of MFP precursors. This is best explained by ectopic Mdka activity in the tailbud, where it drives remaining precursors into the MFP fate. Inhibition of endogenous Mdka in embryos with expanded MFP $(n t)^{-/-}$mutants and cyc-overexpressing embryos) reduced the MFP size, showing that allocation of MFP cells is controlled by trunk-derived Mdka. Thus, in addition to known signals from the shield (and possibly the organizer in higher vertebrates), MFP formation is also controlled by signals from outside these regions.

The epistatic analysis opened the possibility that mdka acts either independently or downstream of factors known to induce MFP during gastrulation. To test whether $m d k a$ is regulated by these factors at the transcriptional level, we analyzed its expression in mutants of the corresponding pathways (Fig. 5). In zebrafish,

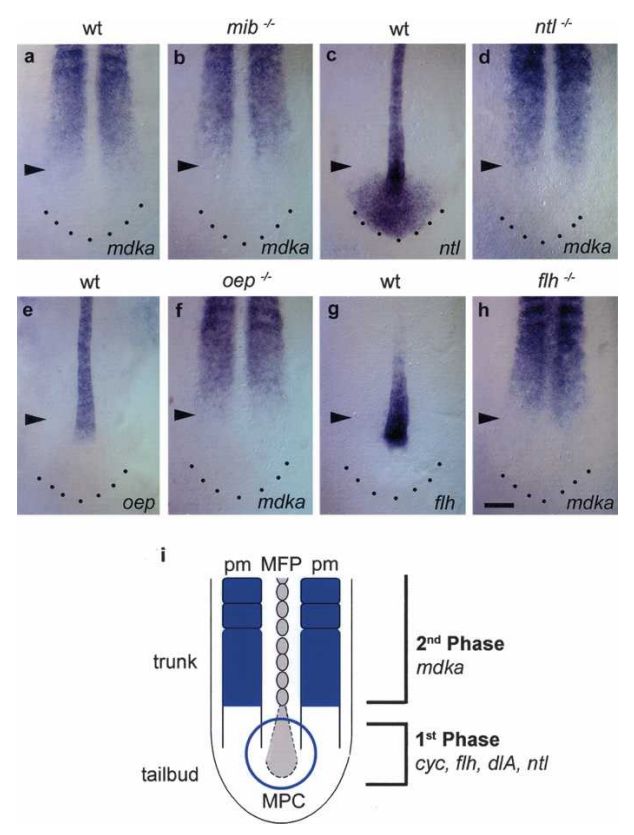

Figure 5. $m d k a$ transcription is not regulated by early acting floor plate-inducing factors. $(a, b)$ In $\mathrm{mib}^{-/-}$embryos, identified by an enlarged notochord and an altered somite shape, mdka expression is normal compared with wild-type embryos $(n=14) .(c, e, g) n t l$, oep, and flh are expressed in the notochord of the trunk and progenitor cells in the tailbud. (c) In addition, $n t l$ is also expressed in the posterior tailbud region. (d) In $n t 1^{-/-}$mutants that lack a notochord and have an enlarged MFP, mdka expression is not impaired $(n=24)$. In these embryos, the paraxial mesoderm is separated at the midline by an expanded MFP. $(f) o_{\text {oep }}^{-1-}$ mutants identified by their characteristic head phenotype form a normal notochord, but lack MFP. In $\mathrm{oep}^{-/-}$, mdka expression is normal $(n=11)$. (h) In $\mathrm{flh}^{-/-}$mutants, $m d k a$ is expressed at the midline $(n=25)$. (i) Two-phase model for MFP formation during neurulation in the zebrafish embryo. Midline precursor cells (MPC) in the tailbud are initially induced to adopt MFP fate in a first Mdka-independent phase. As the embryo extends caudally, Mdka derived from the trunk paraxial mesoderm (pm) regulates allocation of MFP cells (second phase). All pictures are dorsal views of the posterior trunk and tail; anterior is to the top. Arrowheads mark the position of the posterior front of mdka expression in the paraxial mesoderm. Bar: $h, 100 \mu \mathrm{m}$. 
deltaA is expressed in the tailbud and regulates MFP formation within the midline precursor cell population (Appel et al. 1999). In delta/notch-deficient $\mathrm{mib}^{-/-} \mathrm{mu}-$ tants, mdka transcription was not affected (Fig. 5b). At the four-somite stage, $n t l, o e p$, and flh are all expressed in the notochord and its precursors (Fig. $5 \mathrm{c}, \mathrm{e}, \mathrm{g}$ ). In $n t 1^{-/-}$ and oep $^{-/-}$, no effect on mdka expression was observed (Fig. 5d,f). In $\mathrm{flh}^{-1-}$, ectopic mdka expression was evident in the anterior midline mesoderm (Fig. 5h). This, however, does not result from a direct regulation by flh, but rather results from a lack of a notochord in these mutants as notochordal precursors transfate into somitic cells. This consequently leads to the presence of somitic mesoderm at the midline. Despite ectopic mdka expression in the midline of $f l h^{-1-}$ mutants, no expanded MFP was observed. Also, mdka knockdown had no effect on MFP formation in $\mathrm{flh}^{-/-}$(Fig. 4c). This could be explained by the rapid transformation of $f h^{-/-}$midline cells to somitic cells at a stage before Mdka becomes active and can drive these precursors into the MFP fate. Most notably, in all analyzed mutants mdka was not ectopically expressed in the tailbud region, where midline precursor cells are located. Thus, mdka expression appears not to be regulated by factors known as inducers of MFP cells during gastrulation. Our preliminary data suggest that $m d k a$ expression is regulated by Shh. Ectopic overexpression of shh results in a repression of $m d k a$ transcription in the paraxial mesoderm (data not shown). Importantly, it has been shown that Shh is not involved in MFP formation in zebrafish (Schauerte et al. 1998). Consequently, the MFP in shh-overexpressing embryos is normal (data not shown). It therefore is likely that Shh overexpression does not sufficiently inhibit Mdka activity to interfere with MFP formation.

Both epistatic and mutant analyses suggest that $m d k a$ acts independently of known MFP-inducing factors during neurulation. Our data, however, are also consistent with the possibility that Mdka acts by promoting such signals, in particular Cyc. This is supported by the observation that knockdown of mdka in $n t 1^{-/-}$mutants and in cyc-overexpressing embryos reduces the MFP not much further than to wild-type levels. In both situations, sufficient levels of Cyc activity could account for MFP formation. Ectopic Mdka rescues MFP in Nodal-deficient $\mathrm{oep}^{-/-}$mutants. This does not necessarily contradict the idea that Mdka promotes Cyc signaling, as residual maternally derived Nodal components are present in the analyzed zygotic oep mutants. On the other hand, $m d k a$ is not expressed in the shield and tailbud, where MFP-inducing signals like Cyc are active. Rather, Mdka is derived from the trunk region and thus acts in a spatially and temporarily independent manner on the allocation of MFP precursors.

We therefore propose a two-phase model for MFP formation in zebrafish (Fig. 5i). In a first Mdka-independent phase during gastrulation, induction of MFP precursors in the embryonic shield is regulated by Ntl, Cyc, Delta/ Notch, and others (Halpern et al. 1997; Appel et al. 1999; Amacher et al. 2002; Tian et al. 2003). During neurulation, midline precursor cells in the tailbud are still mul- tipotential and form MFP, as well as the notochord and hypochord. Based on our data, we now postulate a second Mdka-dependent phase of MFP formation that becomes important during this period of development. As the embryo elongates along its anteroposterior axis, initially induced precursors come under influence of Mdka expressed within the paraxial mesoderm in a caudally extending wave. This results in the refinement of a subset of precursor cells and their final allocation into the MFP. Our data have shown that both phases are required for complete formation of MFP. These phases appear strictly separated from each other in a temporal and spatial manner.

\section{Conclusion}

Two different models have been used in the past to explain MFP formation in vertebrates (see Strähle et al. 2004). On the one hand, several lines of evidence suggest that MFP is induced in the neural plate during neurulation in chicken and mouse. This is controlled by signals secreted from the underlying notochord, most notably Sonic Hedgehog (Shh) (Placzek et al. 1993; Chiang et al. 1996). Accordingly, floor plate and notochord cells do not share the same origin (Patten et al. 2003; Jeong and Epstein 2003). The alternative model postulates an induction of MFP in the early organizer within a common pool of midline precursor cells that also give rise to the notochord and hypochord (Halpern et al. 1997; Teillet et al. 1998; Appel et al. 1999; Le Douarin and Halpern 2000). Our observations are consistent with an origin of MFP cells from midline precursor cells in zebrafish. In addition to this we show that signals from outside the shield are required for complete MFP formation. This aspect is consistent with the model of trunk-derived signals involved in this process. While these signals are secreted from the notochord in chicken (Placzek et al. 2000), we show an involvement of paraxial mesoderm in zebrafish.

In higher vertebrates, Midkine and the related Pleiotrophin show widespread expression during embryogenesis. In vitro experiments have implicated these growth factors in a variety of processes, including neurite outgrowth and survival, angiogenesis, and tumor growth (for review, see Muramatsu 2002). The exact in vivo function, in particular its role during mammalian MFP formation, however, remains unclear as Midkine knockout mice show no obvious embryonic phenotype (Nakamura et al. 1998). In vitro binding studies identified several candidate receptors for Midkine, including receptor-type protein-tyrosine phosphatase $\zeta$ and LDL receptor-related proteins (LRPs) (for review, see Kadomatsu and Muramatsu 2004). It will be interesting to analyze in the future whether any of these candidate receptors binds to Mdka in vivo and is dynamically expressed in subsets of midline precursor cells during embryonic development.

The identification of Mdka as a novel factor involved in MFP formation is a step forward on the way to elucidate this process in higher vertebrates and allows us to explain species-specific differences.

\section{Materials and methods}

Wild-type and mutant zebrafish stocks

Wild-type fish were reared and embryos obtained as described (Westerfield 1995). The mutant alleles used were floating head $\left(f l h^{n 1}\right)$, mindbomb $\left(\mathrm{mib}^{\text {ta52b}}\right)$, no tail $\left(\mathrm{nt}^{\mathrm{b}} \mathrm{b}^{195}\right)$, and one-eyed-pinhead (oep $\left.{ }^{\text {m134}}\right)$. 
Schäfer et al.

RT-PCR analysis

For analysis of $m d k a$ expression, cDNA was prepared from pooled zebrafish embryos at different stages and used for RT-PCR using primers MK3-105 (5'-CACTCTGCATTTGACTTTCCT-3') and MKEST-UP (5'GTTTCAGTGAGGGAACTTTCG-3'). Actin expression was determined for calibration using the primers actin-up $\left(5^{\prime}\right.$-TTCAACAGCCCT GCCATGTA-3') and actin-down (5'-GCAGCTCATAGCTCTTCTC CAGGGAG- $\left.3^{\prime}\right)$.

RNA in situ hybridization

One- and two-color whole mount in situ hybridizations were performed as described (Winkler et al. 2003). Stained embryos were manually dissected from the yolk and flat mounted in $100 \%$ glycerol for photography. Sections were made manually using razor blades.

\section{Zebrafish embryo injection}

Capped messenger RNA was transcribed in vitro as described (Winkler et al. 2003). Fifty picograms $m d k a$ and $3 \mathrm{pg} c y c$ RNA were injected into embryos at the one- to two-cell stage. For knockdown experiments, embryos were injected with the morpholino mdka MO (5'-CCGCATTTT GTTTTCTGTGTCGAAA-3') at different doses. The most efficient dose (18-20 ng/nL) was determined and the specificity of the oligo confirmed in control experiments (see Supplementary Fig. S4).

\section{Acknowledgments}

We thank R. Dorsky and J.N. Volff for discussions and comments on the manuscript; D. Lamatsch and H. Feldhaar for help with the statistical analyses; and M. Carl, S. Wilson, G. Begemann, B. Appel, R. Köster, and the Zebrafish International Resource Center (NIH-NCRR \#RR12546) for zebrafish mutants and reagents. M. Schäfer is supported by a Boehringer Ingelheim Fonds Ph.D. fellowship, J.W. by HFSPO, M. Schartl by the "Fonds der Chemischen Industrie" and "Sonderforschungsbereich 465," and C.W. by the DFG GRK1048.

\section{References}

Amacher, S.L., Draper, B.W., Summers, B.R., and Kimmel, C.B. 2002. The zebrafish T-box genes no tail and spadetail are required for development of trunk and tail mesoderm and MFP. Development 129: 33113323.

Appel, B., Fritz, A., Westerfield, M., Grunwald, D.J., Eisen, J.S., and Riley B.B. 1999. Delta-mediated specification of midline cell fates in zebrafish embryos. Curr. Biol. 9: 247-256.

Chiang, C., Litingtung, Y., Lee, E., Young, K.E., Corden, J.L., Westphal, H., and Beachy, P.A. 1996. Cyclopia and defective axial patterning in mice lacking Sonic hedgehog gene function. Nature 383: 407-413.

Gritsman, K., Zhang, J., Cheng, S., Heckscher, E., Talbot, W.S., and Schier, A.F. 1999. The EGF-CFC protein one-eyed pinhead is essential for nodal signaling. Cell 97: 121-132.

Halpern, M.E., Hatta, K., Amacher, S.L., Talbot, W.S., Yan, Y.L., Thisse, B., Thisse, C., Postlethwait, J.H., and Kimmel, C.B. 1997. Genetic interactions in zebrafish midline development. Dev. Biol. 187: 154-170.

Itoh, M., Kim, C.H., Palardy, G., Oda, T., Jiang, Y.J., Maust, D., Yeo, S.Y., Lorick, K., Wright, G.J., Ariza-McNaughton, L., et al. 2003. Mind bomb is a ubiquitin ligase that is essential for efficient activation of Notch signaling by Delta. Dev. Cell 4: 67-82.

Jeong, Y. and Epstein, D.J. 2003. Distinct regulators of Shh transcription in the floor plate and notochord indicate separate origins for these tissues in the mouse node. Development 130: 3891-3902.

Kadomatsu, K. and Muramatsu, T. 2004. Midkine and pleiotrophin in neural development and cancer. Cancer Lett. 204: 127-143.

Krauss, S., Concordet, J.P., and Ingham, P.W. 1993. A functionally conserved homolog of the Drosophila segment polarity gene $h h$ is expressed in tissues with polarizing activity in zebrafish embryos. Cell 75: 1431-1444.

Le Douarin, N.M. and Halpern, M.E. 2000. Discussion point. Origin and specification of the neural tube floor plate: Insights from the chick and zebrafish. Curr. Opin. Neurobiol. 10: 23-30.

Lopez, S.L., Paganelli, A.R., Rosato Siri, M.V., Ocana, O.H., Franco, P.G., and Carrasco, A.E. 2003. Notch activates sonic hedgehog and both are involved in the specification of dorsal midline cell-fates in Xenopus. Development 130: 2225-2238.
Matise, M.P., Epstein, D.J., Park, H.L., Platt, K.A., and Joyner, A.L. 1998. Gli2 is required for induction of floor plate and adjacent cells, but not most ventral neurons in the mouse central nervous system. Development 125: 2759-2770.

Muramatsu, T. 2002. Midkine and pleiotrophin: Two related proteins involved in development, survival, inflammation and tumorigenesis. J. Biochem. 132: 359-371.

Nakamura, E., Kadomatsu, K., Yuasa, S., Muramatsu, H., Mamiya, T., Nabeshima, T., Fan, Q.W., Ishiguro, K., Igakura, T., Matsubara, S., et al. 1998. Disruption of the midkine gene (Mdk) resulted in altered expression of a calcium binding protein in the hippocampus of infant mice and their abnormal behaviour. Genes Cells 3: 811-822.

Nasevicius, A. and Ekker, S.C. 2000. Effective targeted gene 'knockdown' in zebrafish. Nat. Genet. 26: 216-220.

Park, H.C., Mehta, A., Richardson, J.S., and Appel, B. 2002. olig2 is required for zebrafish primary motor neuron and oligodendrocyte development. Dev. Biol. 248: 356-368.

Patten, I., Kulesa, P., Shen, M.M., Fraser, S., and Placzek, M. 2003. Distinct modes of floor plate induction in the chick embryo. Development 130: 4809-4821.

Placzek, M., Jessell, T.M., and Dodd, J. 1993. Induction of floor plate differentiation by contact-dependent, homeogenetic signals. Development 117: 205-218.

Placzek, M., Dodd, J., and Jessell, T.M. 2000. Discussion point. The case for floor plate induction by the notochord. Curr. Opin. Neurobiol. 10: $15-22$.

Schäfer, M., Kinzel, D., Neuner, C., Schartl, M., Volff, J.N., and Winkler, C. 2005. Hedgehog and retinoid signalling confines $\mathrm{nkx} 2.2 \mathrm{~b}$ expression to the lateral floor plate of the zebrafish trunk. Mech. Dev. 122: 43-56.

Schauerte, H.E., van Eeden, F.J., Fricke, C., Odenthal, J., Strahle, U., and Haffter, P. 1998. Sonic hedgehog is not required for the induction of MFP cells in the zebrafish. Development 125: 2983-2993.

Strähle, U., Jesuthasan, S., Blader, P., Garcia-Villalba, P., Hatta, K., and Ingham, P.W. 1997. One-eyed pinhead is required for development of the ventral midline of the zebrafish (Danio rerio) neural tube. Genes Funct. 1: 131-148.

Strähle, U., Lam, C.S., Ertzer, R., and Rastegar, S. 2004. Vertebrate floorplate specification: Variations on common themes. Trends Genet. 20: $155-162$.

Teillet, M.A., Lapointe, F., and Le Douarin, N.M. 1998. The relationships between notochord and floor plate in vertebrate development revisited. Proc. Nat1. Acad. Sci. 95: 11733-11738.

Tian, J., Yam, C., Balasundaram, G., Wang, H., Gore, A., and Sampath, K. 2003. A temperature-sensitive mutation in the nodal-related gene cyclops reveals that the floor plate is induced during gastrulation in zebrafish. Development 130: 3331-3342.

Westerfield, M. 1995. The zebrafish book. University of Oregon Press, Eugene, OR.

Winkler, C., Schäfer, M., Duschl, J., Schartl, M., and Volff, J.N. 2003. Functional divergence of two zebrafish midkine growth factors following fish-specific gene duplication. Genome Res. 13: 1067-1081. 


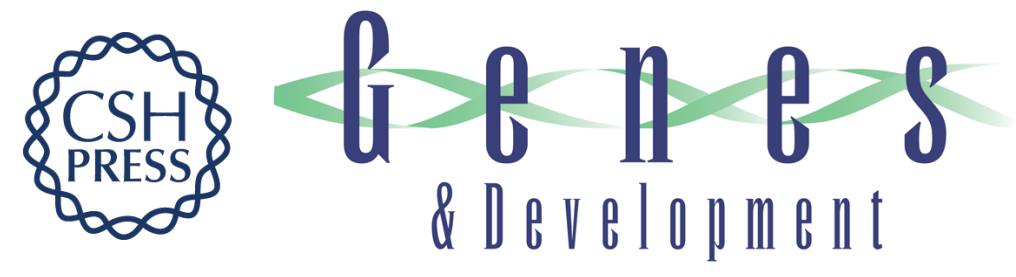

\section{Medial floor plate formation in zebrafish consists of two phases and requires trunk-derived Midkine-a}

Matthias Schäfer, Martina Rembold, Joachim Wittbrodt, et al.

Genes Dev. 2005, 19:

Access the most recent version at doi:10.1101/gad.336305

\section{Supplemental http://genesdev.cshlp.org/content/suppl/2005/04/01/19.8.897.DC1 Material}

References This article cites 25 articles, 9 of which can be accessed free at: http://genesdev.cshlp.org/content/19/8/897.full.html\#ref-list-1

\section{License}

Email Alerting

Receive free email alerts when new articles cite this article - sign up in the box at the top Service 